\title{
The Infinitesimal Spectral Rigidity of the Real Grassmannians of Rank Two
}

\author{
Jacques Gasqui and Hubert Goldschmidt
}

Dedicated to Professor M. Kuranishi on his 70th birthday

\section{Introduction}

Let $(X, g)$ be a Riemannian symmetric space of compact type. Consider a family of Riemannian metrics $\left\{g_{t}\right\}$ on $X$, for $|t|<\varepsilon$, with $g_{0}=g$. We say that $\left\{g_{t}\right\}$ is an isospectral deformation of $g$ if the spectrum of the Laplacian of the metric $g_{t}$ is independent of $t$. We say that the space $(X, g)$ is infinitesimally spectrally rigid (i.e., spectrally rigid to first order) if, for every such isospectral deformation $\left\{g_{t}\right\}$ of $g$, there is a one-parameter family of diffeomorphisms $\varphi_{t}$ of $X$ such that $g_{t}=\varphi_{t}^{*} g$ to first order in $t$ at $t=0$, or equivalently if the infinitesimal deformation $\frac{d}{d t} g_{t \mid t=0}$ of $\left\{g_{t}\right\}$ is a Lie derivative of the metric $g$.

In [13], V. Guillemin proves that the infinitesimal deformation $h$ of an isospectral deformation of $g$ satisfies the following integral condition: for every maximal flat totally geodesic torus $Z$ contained in $X$ and for all parallel vector fields $\zeta$ on $Z$, the integral

$$
\int_{Z} h(\zeta, \zeta) d Z
$$

vanishes, where $d Z$ is the Riemannian measure of $Z$. If all of these integrals corresponding to a symmetric 2-form $h$ on $X$ vanish, we say that $h$ satisfies the Guillemin condition. It is easily verified that a Lie derivative of the metric always satisfies the Guillemin condition. We say that the space $(X, g)$ is rigid in the sense of Guillemin if the following Radon transform property holds on $X$ : the only symmetric 2 -forms on $X$ satisfying the Guillemin condition are the Lie derivatives of the metric $g$. Thus according to [13], if the space $(X, g)$ is rigid in the sense of Guillemin, it is infinitesimally spectrally rigid.

Received December 13, 1995

The second author was supported in part by NSF Grant DMS 92-03974. 
We are interested in determining which irreducible symmetric spaces of compact type are infinitesimally spectrally rigid. Although much work has been done on the problem of isospectrality, there are still very few results for positively curved spaces.

In [9], we study the real Grassmannian $G_{2, n}$ of (unoriented) 2-planes in $\mathbb{R}^{n+2}$, with $n \geq 3$. Here, we present an outline of the proof of the following result of [9]:

Theorem 1. For $n \geq 3$, the real Grassmannian $G_{2, n}$ is rigid in the sense of Guillemin.

The real Grassmannian $G_{2, n}$, which is a space of rank 2, is therefore infinitesimally spectrally rigid. This provides us with the first examples of symmetric spaces (with positive curvature) of rank $>1$ which are infinitesimally spectrally rigid.

All the previously known spectral rigidity results for symmetric spaces with positive curvature concern spaces of rank one. In fact, the Guillemin rigidity of the spaces of rank one which are not spheres was proved by Michel [14] for the real projective spaces $\mathbb{R P}^{n}$, and by Michel [14] and Tsukamoto [18] for the other projective spaces (see also $[2],[5],[6])$. On the other hand, for $2 \leq n \leq 6$, the spectral rigidity of the $n$-sphere $S^{n}$ and the real projective space $\mathbb{R} \mathbb{P}^{n}$ is established by Berger and Tanno (see [1] and [17]).

\section{$\S 1$. The maximal flat Radon transform}

Let $X$ be a manifold whose tangent and cotangent bundles we denote by $T$ and $T^{*}$. By $S^{k} E$ and $\bigwedge^{j} E$, we shall mean the $k$-th symmetric product and the $j$-th exterior product of a vector bundle $E$ over $X$, respectively. If $E$ is a vector bundle over $X$, we denote by $E_{\mathbb{C}}$ its complexification, by $\mathcal{E}$ the sheaf of sections of $E$ over $X$ and by $C^{\infty}(E)$ the space of global sections of $E$ over $X$.

Let $g$ be a Riemannian metric on $X$ and let $\nabla$ be the Levi-Civita connection of $(X, g)$. The Killing operator

$$
D_{0}: \mathcal{T} \rightarrow S^{2} \mathcal{T}^{*}
$$

of $(X, g)$, sending a vector field $\xi$ on $X$ into the Lie derivative of $g$ along $\xi$, and the symmetrized covariant derivative

$$
D^{1}: \mathcal{T}^{*} \rightarrow S^{2} \mathcal{T}^{*}
$$

defined by

$$
\left(D^{1} \theta\right)(\xi, \eta)=\frac{1}{2}((\nabla \theta)(\xi, \eta)+(\nabla \theta)(\eta, \xi)),
$$


for $\theta \in \mathcal{T}^{*}, \xi, \eta \in T$, are related by the formula

$$
\frac{1}{2} D_{0} \xi=D^{1} g^{b}(\xi)
$$

for $\xi \in \mathcal{T}$, where $g^{b}: T \rightarrow T^{*}$ is the isomorphism determined by the metric $g$.

Let $R$ be the Riemann curvature tensor, as defined in [5, §4], which is a section of the bundle $\bigwedge^{2} T^{*} \otimes \bigwedge^{2} T^{*}$, and let $\widetilde{R}$ be the section of $\bigwedge^{2} T^{*} \otimes T^{*} \otimes T$ determined by

$$
g\left(\widetilde{R}\left(\xi_{1}, \xi_{2}, \xi_{3}\right), \xi_{4}\right)=R\left(\xi_{1}, \xi_{2}, \xi_{3}, \xi_{4}\right),
$$

for $\xi_{1}, \xi_{2}, \xi_{3}, \xi_{4} \in T$. Let

$$
D_{g}: S^{2} \mathcal{T}^{*} \rightarrow \bigwedge^{2} \mathcal{T}^{*} \otimes \bigwedge^{2} \mathcal{T}^{*}
$$

be the linear differential operator defined by

$$
\begin{aligned}
&\left(D_{g} h\right)\left(\xi_{1}, \xi_{2}, \xi_{3}, \xi_{4}\right) \\
&=\frac{1}{2}\left\{\left(\nabla^{2} h\right)\left(\xi_{1}, \xi_{3}, \xi_{2}, \xi_{4}\right)+\left(\nabla^{2} h\right)\left(\xi_{2}, \xi_{4}, \xi_{1}, \xi_{3}\right)\right. \\
&-\left(\nabla^{2} h\right)\left(\xi_{1}, \xi_{4}, \xi_{2}, \xi_{3}\right)-\left(\nabla^{2} h\right)\left(\xi_{2}, \xi_{3}, \xi_{1}, \xi_{4}\right) \\
&\left.-h\left(\widetilde{R}\left(\xi_{1}, \xi_{2}, \xi_{3}\right), \xi_{4}\right)+h\left(\widetilde{R}\left(\xi_{1}, \xi_{2}, \xi_{4}\right), \xi_{3}\right)\right\},
\end{aligned}
$$

for $h \in S^{2} \mathcal{T}^{*}$ and $\xi_{1}, \xi_{2}, \xi_{3}, \xi_{4} \in T$.

We now suppose that $(X, g)$ is a Riemannian symmetric space of compact type. We write $X$ as a homogeneous space $G / H$, where $G$ is a compact, connected semi-simple Lie group, which acts on $X$ by isometries, and $H$ is a closed subgroup of $G$ such that $(G, H)$ is a symmetric pair. Since all the maximally totally geodesic flat tori of $X$ are conjugate under the action of $G$ on $X$, the space $\Xi$ of all such tori of $X$ may be regarded as a homogeneous space of $G$. The maximal flat Radon transform of $X$ is a $G$-equivariant linear mapping from the space of functions on $X$ to the space of functions on $\Xi$; it assigns to a function $f$ on $X$ the function $\hat{f}$ on $\Xi$, whose value at a torus $Z$ of $\Xi$, is the integral

$$
\int_{Z} f d Z
$$

of $f$ over $Z$, where $d Z$ is the Riemannian measure of $Z$. Together with Guillemin's above-mentioned result, this leads us to define a maximal flat Radon transform for symmetric 2 -forms as follows. Let $L$ be the 
homogeneous $G$-vector bundle over $\Xi$ whose fiber $L_{Z}$ at a point $Z \in \Xi$ is the space of all parallel vector fields on the flat torus $Z$. We consider the space $C^{\infty}\left(\Xi, S^{2} L^{*}\right)$ of all sections of the vector bundle $S^{2} L^{*}$ (of quadratic forms on $L$ ) over $\Xi$. The maximal flat Radon transform for symmetric 2 -forms on $X$ is the linear mapping

$$
I: C^{\infty}\left(S^{2} T^{*}\right) \rightarrow C^{\infty}\left(\Xi, S^{2} L^{*}\right)
$$

from the space of symmetric 2-forms on $X$ to the space of quadratic forms on $L$, which assigns to a symmetric 2-form $h$ on $X$ the section $I(h)$ of $S^{2} L^{*}$ whose value at the point $Z \in \Xi$ is determined by

$$
I(h)\left(\zeta_{1}, \zeta_{2}\right)=\int_{Z} h\left(\zeta_{1}, \zeta_{2}\right) d Z
$$

where $\zeta_{1}, \zeta_{2}$ are elements of the fiber $L_{Z}$. The vector bundle $S^{2} T^{*}$ is a homogeneous $G$-bundle over $X$ and it is easily verified that the mapping $I$ is $G$-equivariant. Clearly, a symmetric 2-form $h$ on $X$ satisfies the Guillemin condition if and only if $I(h)$ vanishes.

The adjoint space of $X$ is the symmetric space which admits $X$ as a Riemannian cover and which is itself not a Riemannian cover of another symmetric space. For example, the adjoint space of the $n$-sphere $S^{n}$ is the real projective space $\mathbb{R} P^{n}$. The maximal flat tori of $S^{n}$ are the closed geodesics (i.e. the great circles). A function on $\mathbb{R P}^{n}$ lifts to an even function on $S^{n}$, and all the even functions on $S^{n}$ arise in this manner. The kernel of the maximal flat Radon transform of $S^{n}$ is the space of the odd functions on $S^{n}$. In fact, the Radon transform is injective when restricted to the even functions on $S^{n}$; this is equivalent to the classic fact that the Radon transform for functions on $\mathbb{R P}^{n}$ is injective. In [11] and [12], Grinberg generalizes these results and proves that the maximal flat Radon transform for functions on $X$ is injective if and only if the space $X$ is equal to its adjoint space.

We say that a symmetric 2 -form on $X$ satisfies the zero-energy condition if all its integrals over the closed geodesics of $X$ vanish. Lie derivatives of the metric always satisfy this condition. The space $(X, g)$ is said to be infinitesimally rigid if the only symmetric 2 -forms on $X$ satisfying the zero-energy condition are the Lie derivatives of the metric $g$. For spaces of rank one, this notion of infinitesimal rigidity coincides with rigidity in the sense of Guillemin. Thus, as stated in the introduction, the infinitesimal rigidity of the projective spaces which are not spheres has been established.

In $[9, \S 1]$, we show that: 
Lemma 1. A symmetric 2-form on $X$ satisfying the zero-energy condition also satisfies the Guillemin condition.

\section{$\S 2$. The complex quadrics and the real Grassmannians}

Let $n$ be an integer $\geq 3$. We henceforth suppose that $X$ is the complex quadric $Q_{n}$, which is the complex hypersurface of complex projective space $\mathbb{C P}^{n+1}$ defined by the homogeneous equation

$$
\zeta_{0}^{2}+\zeta_{1}^{2}+\cdots+\zeta_{n+1}^{2}=0
$$

where $\zeta=\left(\zeta_{0}, \zeta_{1}, \ldots, \zeta_{n+1}\right)$ is the standard complex coordinate system of $\mathbb{C}^{n+2}$. Let $g$ be the Kähler metric on $Q_{n}$ induced by the FubiniStudy metric on $\mathbb{C P}^{n+1}$ of constant holomorphic curvature 4 . The group $S U(n+2)$ acts on $\mathbb{C P}^{n+1}$ by holomorphic isometries. Its subgroup $S O(n+2)$ leaves the submanifold $Q_{n}$ of $\mathbb{C P}^{n+1}$ invariant and acts transitively on $Q_{n}$; it is then easily verified that $Q_{n}$ is the homogeneous space

$$
S O(n+2) / S O(2) \times S O(n)
$$

of the group $S O(n+2)$, which is an irreducible Hermitian symmetric space of compact type of rank 2 .

We consider the functions

$$
\begin{aligned}
& f_{0,1}(\zeta)=\left(\zeta_{0}+i \zeta_{1}\right)\left(\bar{\zeta}_{2}+i \bar{\zeta}_{3}\right)-\left(\zeta_{2}+i \zeta_{3}\right)\left(\bar{\zeta}_{0}+i \bar{\zeta}_{1}\right) \\
& f_{1,0}(\zeta)=\left(\zeta_{0}+i \zeta_{1}\right)\left(\bar{\zeta}_{0}+i \bar{\zeta}_{1}\right)
\end{aligned}
$$

on $\mathbb{C}^{n+2}$. If $r, s$ are integers $\geq 0$, the function $f_{r, s}=f_{1,0}^{r} \cdot f_{0,1}^{s}$ on $\mathbb{C}^{n+2}$ is invariant under $U(1)$; its restriction to the unit sphere $S^{2 n+3}$ of $\mathbb{C}^{n+2}$ induces by passage to the quotient a function on $\mathbb{C} \mathbb{P}^{n+1}$, whose restriction to $Q_{n}$ we denote by $\tilde{f}_{r, s}$.

The complex conjugation of $\mathbb{C}^{n+2}$ induces an involutive isometry of $\mathbb{C P}^{n+1}$ which preserves the quadric $Q_{n}$. The induced isometry $\tau$ of $Q_{n}$ commutes with the action of the group $S O(n+2)$. The group $\{\mathrm{id}, \tau\}$ of isometries of $Q_{n}$ acts freely on $Q_{n}$ and so we may consider the quotient Riemannian manifold $Y$ of $X$ by this group, with the metric induced by $g$, which is also a homogeneous space of $S O(n+2)$. The natural projection $\varpi: Q_{n} \rightarrow Y$ is a Riemannian submersion and a two-fold covering.

Let $\widetilde{G}_{2, n}$ be the real Grassmannian of oriented 2-planes in $\mathbb{R}^{n+2}$. It is easily verified that the mapping

$$
\Psi: \widetilde{G}_{2, n} \rightarrow \mathbb{C P}^{n+1}
$$


sending the oriented 2-plane of $\mathbb{R}^{n+2}$ determined by $x \wedge y$, where $\{x, y\}$ is an orthonormal system of vectors of $\mathbb{R}^{n+2}$, into the point of $\mathbb{C P} \mathbb{P}^{n+1}$ with homogeneous coordinates $\zeta=x+i y \in \mathbb{C}^{n+2}$, and that its image is contained in $Q_{n}$. This mapping $\Psi$ allows us to identify the quadric $Q_{n}$ with $\widetilde{G}_{2, n}$. If $\tau_{0}$ is the involution of $\widetilde{G}_{2, n}$ corresponding to the change of orientation of a 2-plane, we see that

$$
\tau \circ \Psi=\Psi \circ \tau_{0} .
$$

Thus we may identify $Y$ with the real Grassmannian $G_{2, n}$ and $\varpi$ with the natural projection of $\widetilde{G}_{2, n}$ onto $G_{2, n}$, and then view the quadric $Q_{n}$ as the double cover of the real Grassmannian $G_{2, n}$. In fact, the adjoint space of $Q_{n}$ is the Grassmannian $G_{2, n}$.

This situation is entirely analogous to that of the sphere $S^{n}$ viewed as the double cover of the real projective space $\mathbb{R P}^{n}$. If we consider the sphere $S^{n}$ as the space of oriented lines in $\mathbb{R}^{n+1}$ and the real projective space $\mathbb{R P}^{n}$ as the set of lines in $\mathbb{R}^{n+1}$, the antipodal mapping $\sigma$ of the sphere corresponds to the change of orientation of a line and the projective space $\mathbb{R} \mathbb{P}^{n}$ is equal to the quotient of $S^{n}$ by the group $\{\mathrm{id}, \sigma\}$ of isometries of $S^{n}$.

By analogy with the sphere, the involution $\tau$ of $Q_{n}$ determines notions of even and odd tensors on $Q_{n}$ : a symmetric $p$-form $\theta$ on $Q_{n}$ is said to be even (resp. odd) if $\tau^{*} \theta=\theta$ (resp. $\tau^{*} \theta=-\theta$ ). The function $\tilde{f}_{r, s}$ on $Q_{n}$ is even (resp. odd) if and only if the integer $s$ is even (resp. odd). The space $C^{\infty}\left(S^{p} T_{\mathbb{C}}^{*}\right)^{\text {ev }}\left(\right.$ resp. $\left.C^{\infty}\left(S^{p} T_{\mathbb{C}}^{*}\right)^{\text {odd }}\right)$ of all even (resp. odd) complex symmetric $p$-forms on $X$ is an $S O(n+2)$-submodule of $C^{\infty}\left(S^{p} T_{\mathbb{C}}^{*}\right)$, and we have the decomposition

$$
C^{\infty}\left(S^{p} T_{\mathbb{C}}^{*}\right)=C^{\infty}\left(S^{p} T_{\mathbb{C}}^{*}\right)^{\mathrm{ev}} \oplus C^{\infty}\left(S^{p} T_{\mathbb{C}}^{*}\right)^{\text {odd }}
$$

We now construct an explicit maximal totally geodesic flat torus of $Q_{n}$. Let

$$
\pi: \mathbb{C}^{n+2}-\{0\} \rightarrow \mathbb{C P}^{n+1}
$$

be the natural projection. We consider the submanifold $Z_{0}$ of $X$ which is the image of the mapping $\sigma: \mathbb{R}^{2} \rightarrow X$ defined by $\sigma(\theta, \varphi)=\pi \tilde{\sigma}(\theta, \varphi)$, where

$$
\tilde{\sigma}(\theta, \varphi)=(\cos \theta, \sin \theta, 0, \ldots, 0,-i \sin \varphi, i \cos \varphi) \in \mathbb{C}^{n+2},
$$

for $(\theta, \varphi) \in \mathbb{R}^{2}$. This mapping $\sigma$ satisfies

$$
\sigma(\theta, \varphi)=\sigma(\theta+2 k \pi, \varphi+2 l \pi)=\sigma(\theta+k \pi, \varphi+k \pi),
$$


for all $k, l \in \mathbb{Z}$ and $(\theta, \varphi) \in \mathbb{R}^{2}$. We consider the group of translations $\Gamma$ of $\mathbb{R}^{2}$ generated by the vectors $(2 \pi, 0)$ and $(\pi, \pi)$ and the flat torus $\mathbb{R}^{2} / \Gamma$, which is the quotient of $\mathbb{R}^{2}$ by the group $\Gamma$. According to the preceding relations, we see that $\sigma$ induces an imbedding

$$
\bar{\sigma}: \mathbb{R}^{2} / \Gamma \rightarrow X .
$$

Let $(\theta, \varphi)$ be the standard coordinate system on $\mathbb{R}^{2}$. It is easily verified that

$$
\sigma^{*} g=\frac{1}{2}(d \theta \otimes d \theta+d \varphi \otimes d \varphi) .
$$

Therefore, if the quotient $\mathbb{R}^{2} / \Gamma$ is endowed with the flat metric induced by the metric $\frac{1}{2}(d \theta \otimes d \theta+d \varphi \otimes d \varphi)$ on $\mathbb{R}^{2}$, the mapping $\bar{\sigma}$ is a totally geodesic isometric imbedding. Thus $Z_{0}$ is a maximal flat totally geodesic torus of $X$.

The vector fields $\partial / \partial \theta$ and $\partial / \partial \varphi$ on $\mathbb{R}^{2}$ are $\sigma$-projectable; thus, there exist well-defined parallel vector fields $\xi_{0}$ and $\eta_{0}$ on $Z_{0}$ determined by $\partial / \partial \theta$ and $\partial / \partial \varphi$, respectively. In fact, $\left\{\xi_{0}, \eta_{0}\right\}$ is a basis for the space of parallel vector fields on $Z_{0}$.

Since the group $S O(n+2)$ acts transitively on $\Xi$, a symmetric 2form $h$ on $X$ satisfies the Guillemin condition if and only if, for all $\phi \in S O(n+2)$, the section $I\left(\phi^{*} h\right)$ of $S^{2} L^{*}$ vanishes at the torus $Z_{0}$ of $\Xi$, or equivalently if

$$
\int_{Z_{0}}\left(\phi^{*} h\right)\left(\xi_{0}, \xi_{0}\right) d Z_{0}=\int_{Z_{0}}\left(\phi^{*} h\right)\left(\eta_{0}, \eta_{0}\right) d Z_{0}=\int_{Z_{0}}\left(\phi^{*} h\right)\left(\xi_{0}, \eta_{0}\right) d Z_{0}=0,
$$

for all $\phi \in S O(n+2)$.

In $[9, \S 3]$, by means of this torus $Z_{0}$ we prove that:

Proposition 1. (i) The odd symmetric 2-forms on $X$ satisfy the Guillemin condition.

(ii) $A$ symmetric 2-form $h$ on $Y$ satisfies the Guillemin condition if and only if the even symmetric 2 -form $\varpi^{*} h$ on $X$ satisfies the Guillemin condition.

On the symmetric space $Q_{n}$, according to $[4, \S 7]$ the Lie derivatives of the metric can be characterized as the symmetric 2 -forms $h$ satisfying the condition $Q_{g} h=0$, where $Q_{g}$ is a non-trivial differential operator of order 3. Let $x_{0}$ be a given point of $X$; choose an open subset $U$ of $X$ containing $x_{0}$ such that $U \cap \tau(U)=\emptyset$. We now choose a symmetric 2-form on $X$ whose support is contained in $U$ and which satisfies $\left(Q_{g} h\right)\left(x_{0}\right) \neq 0$. We know that $h$ is not a Lie derivative of the metric on any neighborhood of $x_{0}$. The symmetric 2 -form $h-\tau^{*} h$ on $X$ is odd and its restriction to $U$ is equal to $h$. Thus we have proved the following: 
Proposition 2. There exist odd symmetric 2-forms on $Q_{n}$ which are not Lie derivatives of the metric.

From the preceding proposition, it follows that the quadric $Q_{n}$ is not is rigid in the sense of Guillemin. This is analogous to the non-injectivity of the maximal flat Radon transform (for functions) on this space.

In order to prove the Guillemin rigidity of the adjoint space $G_{2, n}$ of the quadric $Q_{n}$, according to Proposition 1,(ii) it suffices to verify:

Theorem 2. For $n \geq 3$, an even symmetric 2 -form on $Q_{n}$ satisfies the Guillemin condition if and only if it is the Lie derivative of the metric.

From the infinitesimal rigidity of the quadrics $Q_{n}$, with $n \geq 4$, which we proved in [6], [7] and [8], we infer that it suffices to prove the preceding theorem when $n=3$. Indeed, let $h$ be an even symmetric 2 -form on $Q_{n}$, with $n \geq 4$, satisfying the Guillemin condition. Let $\gamma$ be a closed geodesic of $Q_{n}$. It is contained in a totally geodesic submanifold $X^{\prime}$ of $Q_{n}$ isometric to the quadric $Q_{3}$. The restriction of $h$ to $X^{\prime}$ is even and satisfies the Guillemin condition. (The demonstration of these last two assertions is given in the course of the proof of Theorem 10.1 of [9].) Then Theorem 2, with $n=3$, tells us that the restriction of $h$ to $X^{\prime}$ is a Lie derivative of the metric; thus the integral of $h$ over $\gamma$ vanishes. Hence the 2-form $h$ on $Q_{n}$ satisfies the zero-energy condition. The infinitesimal rigidity of the quadric $Q_{n}$ now implies that $h$ is a Lie derivative of the metric.

\section{$\S 3 . \quad$ Totally geodesic spheres}

We now introduce decompositions of the bundle $S^{2} T^{*}$ of symmetric 2 -forms on $X$. The complex structure of $X$ induces a decomposition

$$
S^{2} T^{*}=\left(S^{2} T^{*}\right)^{+} \oplus\left(S^{2} T^{*}\right)^{-}
$$

of the bundle $S^{2} T^{*}$ of symmetric 2 -forms on $X$, where $\left(S^{2} T^{*}\right)^{+}$is the sub-bundle of Hermitian forms and $\left(S^{2} T^{*}\right)^{-}$is the sub-bundle of skewHermitian forms. We now use the differential geometry of $X$ considered as a complex hypersurface of $\mathbb{C P}^{n+1}$, which has been studied by Smyth [15]. The components of the second fundamental form of $X$ in $\mathbb{C} \mathbb{P}^{n+1}$ generate a sub-bundle $E$ of $\left(S^{2} T^{*}\right)^{-}$of rank 2 and determine an involution of the bundle $\left(S^{2} T^{*}\right)^{+}$. If $\left(S^{2} T^{*}\right)^{++}$and $\left(S^{2} T^{*}\right)^{+-}$are the eigenbundles corresponding to the eigenvalues +1 and -1 , respectively, of this involution, we therefore obtain a direct sum decomposition

$$
\left(S^{2} T^{*}\right)^{+}=\left(S^{2} T^{*}\right)^{++} \oplus\left(S^{2} T^{*}\right)^{+-}
$$


of $\left(S^{2} T^{*}\right)^{+}$.

We consider the totally geodesic spheres of the quadric $X$ of dimension 2 which are totally real. According to [3], these spheres have constant curvature 2 and are all conjugate under the action of the group $S O(n+2)$. Moreover, such a sphere is the double cover of a closed totally geodesic submanifold of the real Grassmannian $Y=G_{2, n}$ isometric to the projective plane $\mathbb{R} \mathbb{P}^{2}$ with its metric of constant curvature 2 .

Let $B$ be the sub-bundle of $S^{2} T^{*}$ consisting of the elements of $S^{2} T^{*}$, which vanish when restricted to the closed totally geodesic submanifolds of $X$ isometric to the sphere $S^{2}$ with its metric of constant curvature 2. In $[9, \S 5]$, we verify the following:

Lemma 2. We have

$$
B=\left(S^{2} T^{*}\right)^{+-} .
$$

From Lemma 2 and properties of the operator $D_{g}$, in [9, Lemma 5.2] we deduce that:

Lemma 3. Let $Z$ be a totally geodesic closed submanifold of $X$ isometric to the 2-sphere $S^{2}$ with its metric of constant curvature 2. Let $x \in Z$ and let $\xi_{1}, \xi_{2}, \xi_{3}, \xi_{4} \in T_{x}$ be tangent to $Z$. If $h$ is a section of $\left(S^{2} T^{*}\right)^{+-}$over $X$, then we have

$$
\left(D_{g} h\right)\left(\xi_{1}, \xi_{2}, \xi_{3}, \xi_{4}\right)=0
$$

Using the injectivity of the Radon transform on the real projective plane, in [9, Lemma 5.3] we obtain the following result, which provides us with another relation between our two notions of rigidity:

Lemma 4. Let $h$ be a symmetric 2-form on the real Grassmannian $Y$ satisfying the Guillemin condition. Then the restriction of $h$ to a closed totally geodesic submanifold of $Y$ isometric to the real projective plane with its metric of constant curvature 2 satisfies the zero-energy condition.

From Lemma 4 and the infinitesimal rigidity of the real projective plane (see [14], [2] and [10, §2]), we immediately deduce:

Proposition 3. Let h be a symmetric 2-form on $Y$ satisfying the Guillemin condition and let $Y^{\prime}$ be a closed totally geodesic submanifold of $Y$ isometric to the real projective plane with its metric of constant 
curvature 2. Then the restriction of $h$ to $Y^{\prime}$ is a Lie derivative of the metric on $Y^{\prime}$.

This last proposition together with properties of the operator $D_{g}$ can be used to show the following (see Proposition 5.4 of [9]):

Proposition 4. Let $Z$ be a totally geodesic closed submanifold of $X$ isometric to the 2-sphere $S^{2}$ with its metric of constant curvature 2 . Let $x \in Z$ and let $\xi_{1}, \xi_{2}, \xi_{3}, \xi_{4} \in T_{x}$ be tangent to $Z$. An even symmetric 2 -form $h$ on $X$ which satisfies the Guillemin condition also verifies the equation

$$
\left(D_{g} h\right)\left(\xi_{1}, \xi_{2}, \xi_{3}, \xi_{4}\right)=0
$$

\section{$\S 4$. The Radon transform on the complex quadric}

As we have seen, the mapping $I$ is $S O(n+2)$-equivariant. Thus the kernel of $I$ is an $S O(n+2)$-submodule of $C^{\infty}\left(S^{2} T^{*}\right)$, and the space $\mathcal{N}_{\mathbb{C}}$ consisting of all sections of $S^{2} T_{\mathbb{C}}^{*}$ over $X$ satisfying the Guillemin condition is a $S O(n+2)$-submodule of $C^{\infty}\left(S^{2} T_{\mathbb{C}}^{*}\right)$. The space of all even complex symmetric 2-forms on $X$ satisfying the Guillemin condition is the $S O(n+2)$-submodule

$$
\mathcal{N}_{\mathbb{C}}^{\mathrm{ev}}=\mathcal{N}_{\mathbb{C}} \cap C^{\infty}\left(S^{2} T_{\mathbb{C}}^{*}\right)^{\mathrm{ev}}
$$

of $C^{\infty}\left(S^{2} T_{\mathbb{C}}^{*}\right)$

Let $\Gamma$ be the set of equivalence classes of irreducible $S O(n+2)$ modules over $\mathbb{C}$. The vector bundle $F=S^{p} T_{\mathbb{C}}^{*}$, endowed with the Hermitian scalar product induced by the metric $g$, is homogeneous and unitary. The space $C^{\infty}(F)$ endowed with the Hermitian scalar product obtained from the Hermitian scalar product of $F$ and the $S O(n+2)$ invariant Riemannian measure $d X$ of $X$ is a unitary $S O(n+2)$-module. We denote by $C_{\gamma}^{\infty}(F)$ the isotypic component of this $S O(n+2)$-module $C^{\infty}(F)$ corresponding to $\gamma \in \Gamma$.

The differential operator $D^{1}: \mathcal{T}_{\mathbb{C}}^{*} \rightarrow S^{2} \mathcal{T}_{\mathbb{C}}^{*}$ is homogeneous, and so we have

$$
D^{1} C_{\gamma}^{\infty}\left(T_{\mathbb{C}}^{*}\right) \subset C_{\gamma}^{\infty}\left(S^{2} T_{\mathbb{C}}^{*}\right),
$$

for all $\gamma \in \Gamma$. Since $\tau$ is an isometry and as the Lie derivatives of the metric satisfy the Guillemin condition, we see that

$$
D^{1} C^{\infty}\left(T_{\mathbb{C}}^{*}\right)^{\text {ev }} \subset \mathcal{N}_{\mathbb{C}}^{\text {ev }}
$$

For $\gamma \in \Gamma$, we consider the $S O(n+2)$-submodule

$$
\mathcal{N}_{\gamma}^{\mathrm{ev}}=\mathcal{N}_{\mathbb{C}}^{\mathrm{ev}} \cap C_{\gamma}^{\infty}\left(S^{2} T_{\mathbb{C}}^{*}\right)
$$


of the isotypic component $C_{\gamma}^{\infty}\left(S^{2} T_{\mathbb{C}}^{*}\right)$.

Using the $S O(n+2)$-equivariance of the mapping $I$, the ellipticity of the operator $D^{1}$ and the results of $[20, \S 5]$, we are able to reduce the assertion of Theorem 2 to a question involving the isotypic components of the $S O(n+2)$-module $C^{\infty}\left(S^{2} T_{\mathbb{C}}^{*}\right)^{\text {ev }}$ as follows:

Proposition 5. The even symmetric 2-forms on $Q_{n}$ satisfying the Guillemin condition are Lie derivatives of the metric if and only if the equality

$$
\mathcal{N}_{\gamma}^{\mathrm{ev}}=D^{1} C_{\gamma}^{\infty}\left(T_{\mathbb{C}}^{*}\right)^{\mathrm{ev}}
$$

holds for all $\gamma \in \Gamma$.

In $[9, \S 7]$, we consider an orthonormal basis $\left\{v_{1}, v_{2}, v_{3}, w_{1}, w_{2}, w_{3}\right\}$ of the tangent space $T_{a_{0}}$ at a specific point $a_{0} \in X$ which has the following property: for all $1 \leq i<j \leq 3$, the vectors $\left\{v_{i}, v_{j}\right\}$ (resp. $\left\{w_{i}, w_{j}\right\}$ ) are tangent to a totally geodesic closed submanifold of $X$ isometric to the 2 -sphere $S^{2}$ with its metric of constant curvature 2 . We set

$$
A_{i j} h=\left(D_{g} h\right)\left(v_{i}, v_{j}, v_{i}, v_{j}\right), \quad A_{i j}^{\prime} h=\left(D_{g} h\right)\left(w_{i}, w_{j}, w_{i}, w_{j}\right),
$$

for $1 \leq i<j \leq 3$. Let

$$
D: C^{\infty}\left(S^{2} T_{\mathbb{C}}^{*}\right) \rightarrow \mathbb{C}^{5}
$$

be the linear mapping defined by

$$
D h=\left(A_{12} h, A_{23} h, A_{12}^{\prime} h, A_{13}^{\prime} h, A_{23}^{\prime} h\right),
$$

for $h \in C^{\infty}\left(S^{2} T_{\mathbb{C}}^{*}\right)$. According to Proposition 4, the subspace $\mathcal{N}_{\mathbb{C}}^{\text {ev }}$ of $C^{\infty}\left(S^{2} T_{\mathbb{C}}^{*}\right)$ is contained in the kernel of $D$.

\section{§5. The complex quadric of dimension three}

In this section, we suppose that $n=3$ and that $X$ is the quadric $Q_{3}$ of dimension three.

We denote by $\mathfrak{g}$ the Lie algebra of the group $S O(5)$. We fix a specific Cartan subalgebra $\mathfrak{t}_{\mathbb{C}}$ of the complexification $\mathfrak{g}_{\mathbb{C}}$ of the Lie algebra $\mathfrak{g}$ and consider the linear forms $\lambda_{0}$ and $\lambda_{1}$ on $\mathfrak{t}_{\mathbb{C}}$ defined in $[9, \S 9]$. We choose a Weyl chamber of $\left(\mathfrak{g}_{\mathbb{C}}, \mathfrak{t}_{\mathbb{C}}\right)$ for which the system of positive roots $\Delta^{+}$ of $\mathfrak{g}_{\mathbb{C}}$ is equal to

$$
\lambda_{0}-\lambda_{1}, \quad \lambda_{0}+\lambda_{1}, \quad \lambda_{0}, \quad \lambda_{1} ;
$$

then $\left\{\lambda_{0}-\lambda_{1}, \lambda_{1}\right\}$ is a system of simple roots of $\mathfrak{g}_{\mathbb{C}}$. The highest weight of an irreducible $S O(5)$-module is a linear form $\Lambda=c_{0} \lambda_{0}+c_{1} \lambda_{1}$ on $\mathfrak{t}_{\mathbb{C}}$, 
where $c_{0}, c_{1}$ are integers satisfying $c_{0} \geq c_{1} \geq 0$. The equivalence class of such an $S O(5)$-module is determined by this weight. We identify $\Gamma$ with the set of all such linear forms on $\mathfrak{t}_{\mathbb{C}}$. In particular, we consider the elements

$$
\gamma_{r, s}=(2 r+s) \lambda_{0}+s \lambda_{1}, \quad \gamma_{r, s}^{\prime}=(2 r+s+1) \lambda_{0}+s \lambda_{1}
$$

of $\Gamma$, with $r, s \geq 0$. If $\gamma \in \Gamma$, an $S O(5)$-submodule $W$ of $C_{\gamma}^{\infty}\left(S^{p} T_{\mathbb{C}}^{*}\right)$ is isomorphic to $k$ copies of an irreducible $S O(5)$-module with highest weight $\gamma$, where $k$ is an integer called the multiplicity of $W$ and denoted by Mult $W$. In fact, the multiplicity of $W$ is equal to the dimension of the weight subspace $\mathcal{C}(W)$ of $W$ corresponding to the weight $\gamma$.

The decomposition of the bundle $S^{2} T_{\mathbb{C}}^{*}$ of complex symmetric 2forms on $Q_{3}$ into irreducible $S O(5)$-invariant sub-bundles described in [7] and the branching law of [19] allow us to compute the multiplicities of the isotypic components of $C^{\infty}\left(T_{\mathbb{C}}^{*}\right)$ and $C^{\infty}\left(S^{2} T_{\mathbb{C}}^{*}\right)$, which are given by Proposition 9.1 of [9]. In particular, we obtain:

Proposition 6. Let $\gamma$ be an element of $\Gamma$. If the $S O(5)$-module $C_{\gamma}^{\infty}\left(T_{\mathbb{C}}^{*}\right)\left(\right.$ resp. $\left.C_{\gamma}^{\infty}\left(S^{2} T_{\mathbb{C}}^{*}\right)\right)$ is non-zero, then we may write $\gamma$ in the form $\gamma=\gamma_{r, s}$ or $\gamma=\gamma_{r, s}^{\prime}$, for some integers $r, s \geq 0$.

If we define integers $d_{r, s}$ and $d_{r, s}^{\prime}$ by

$$
d_{r, s}=\left\{\begin{array}{l}
0 \text { if } r=s=0, \\
2 \text { if } r, s \geq 1, \\
1 \text { otherwise }
\end{array} \quad d_{r, s}^{\prime}= \begin{cases}1 & \text { if } s \geq 1 \\
0 & \text { otherwise }\end{cases}\right.
$$

for $r, s \geq 0$, then Proposition 9.1 of [9] tells us that

$$
\begin{aligned}
\operatorname{Mult} C_{\gamma_{r, s}}^{\infty}\left(T_{\mathbb{C}}^{*}\right) & =2 d_{r, s}, \quad \operatorname{Mult} C_{\gamma_{r, s}^{\prime}}^{\infty}\left(T_{\mathbb{C}}^{*}\right)=2 d_{r, s}^{\prime}, \\
\operatorname{Mult} C_{\gamma_{r, s}}^{\infty}\left(S^{2} T_{\mathbb{C}}^{*}\right) & \leq 13, \quad \operatorname{Mult} C_{\gamma_{r, s}^{\prime}}^{\infty}\left(S^{2} T_{\mathbb{C}}^{*}\right) \leq 8,
\end{aligned}
$$

for all $r, s \geq 0$.

According to Strichartz [16], the function $\tilde{f}_{r, s}$ is the highest weight vector of the irreducible $S O(5)$-module $C_{\gamma_{r, s}}^{\infty}(X)=C_{\gamma_{r, s}}^{\infty}\left(S^{0} T_{\mathbb{C}}^{*}\right)$ and an eigenfunction of the Laplacian of $X$, for $r, s \geq 0$. In [9], we use this result together with the equalities (3) to construct explicit highest weight vectors of the $S O(5)$-modules $C_{\gamma_{r, s}}^{\infty}\left(T_{\mathbb{C}}^{*}\right)$ and $C_{\gamma_{r, s}^{\prime}}^{\infty}\left(T_{\mathbb{C}}^{*}\right)$ and to prove the following two lemmas. 
Lemma 5. The multiplicity of the $S O(5)$-module $C_{\gamma_{r, s}}^{\infty}\left(T_{\mathbb{C}}^{*}\right)^{\mathrm{ev}}$ or of the $S O(5)$-module $C_{\gamma_{r, s}}^{\infty}\left(T_{\mathbb{C}}^{*}\right)^{\text {odd }}$ is equal to $d_{r, s}$. Moreover, the kernel of the operator $D^{1}: C^{\infty}\left(T_{\mathbb{C}}^{*}\right) \rightarrow C^{\infty}\left(S^{2} T_{\mathbb{C}}^{*}\right)$ is equal to $C_{\gamma_{0,1}}^{\infty}\left(T_{\mathbb{C}}^{*}\right)^{\mathrm{ev}}$.

Lemma 6. For $r \geq 0, s \geq 1$, the $S O(5)$-modules $C_{\gamma_{r, s}^{\prime}}^{\infty}\left(T_{\mathbb{C}}^{*}\right)^{\text {ev }}$ and $C_{\gamma_{r, s}^{\prime}}^{\infty}\left(T_{\mathbb{C}}^{*}\right)^{\text {odd }}$ are irreducible.

Thus from Proposition 5 and the two preceding lemmas, we deduce:

Proposition 7. The even symmetric 2-forms on $Q_{3}$ satisfying the Guillemin condition are Lie derivatives of the metric if and only if the equalities

$$
\text { Mult } \mathcal{N}_{\gamma_{r, s}}^{\mathrm{ev}} \leq d_{r, s}, \quad \text { Mult } \mathcal{N}_{\gamma_{r, s}^{\prime}}^{\mathrm{ev}} \leq d_{r, s}^{\prime}
$$

hold for all $r, s \geq 0$, and if

$$
\mathcal{N}_{\gamma_{0,1}}^{\mathrm{ev}}=\{0\}
$$

In [9], we introduce finite-dimensional subspaces $V_{r, s}$ and $W_{r, s}$ (resp. $V_{r, s}^{\prime}$ and $\left.W_{r, s}^{\prime}\right)$ of $C_{\gamma_{r, s}}^{\infty}\left(S^{2} T_{\mathbb{C}}^{*}\right)\left(\right.$ resp. $\left.C_{\gamma_{r, s}^{\prime}}^{\infty}\left(S^{2} T_{\mathbb{C}}^{*}\right)\right)$ all of whose elements are vectors of weight $\gamma_{r, s}$ (resp. $\gamma_{r, s}^{\prime}$ ). We define generators of these subspaces in terms of the functions $\tilde{f}_{r, s}$ and explicit Hermitian symmetric 2 -forms on $\mathbb{C P}^{4}$, and then verify that they are either odd or even and that

$$
\begin{aligned}
V_{r, 2 p} & \subset C^{\infty}\left(S^{2} T_{\mathbb{C}}^{*}\right)^{\mathrm{ev}}, & V_{r, 2 p+1} & \subset C^{\infty}\left(S^{2} T_{\mathbb{C}}^{*}\right)^{\text {odd }}, \\
W_{r, 2 p+1} & \subset C^{\infty}\left(S^{2} T_{\mathbb{C}}^{*}\right)^{\mathrm{ev}}, & W_{r, 2 p} & \subset C^{\infty}\left(S^{2} T_{\mathbb{C}}^{*}\right)^{\text {odd }}, \\
V_{r, 2 p}^{\prime} & \subset C^{\infty}\left(S^{2} T_{\mathbb{C}}^{*}\right)^{\mathrm{ev}}, & V_{r, 2 p+1}^{\prime} & \subset C^{\infty}\left(S^{2} T_{\mathbb{C}}^{*}\right)^{\text {odd }}, \\
W_{r, 2 p+1}^{\prime} & \subset C^{\infty}\left(S^{2} T_{\mathbb{C}}^{*}\right)^{\mathrm{ev}}, & W_{r, 2 p}^{\prime} & \subset C^{\infty}\left(S^{2} T_{\mathbb{C}}^{*}\right)^{\text {odd }},
\end{aligned}
$$

for all $r, p \geq 0$.

In $[9, \S 7$ and $\S 9]$, we simultaneously determine the dimension of the spaces $V_{r, s}, W_{r, s}, V_{r, s}^{\prime}$ and $W_{r, s}^{\prime}$ and prove the following lemma:

Lemma 7. Let $r, s \geq 0$ be given integers.

(i) If $s$ is even, we have

$$
\operatorname{dim}\left(\mathcal{N}_{\mathbb{C}} \cap V_{r, s}\right) \leq d_{r, s}, \quad \operatorname{dim}\left(\mathcal{N}_{\mathbb{C}} \cap V_{r, s}^{\prime}\right) \leq 1 .
$$

(ii) If $s$ is odd, we have

$$
\operatorname{dim}\left(\mathcal{N}_{\mathbb{C}} \cap W_{r, s}\right) \leq d_{r, s}, \quad \operatorname{dim}\left(\mathcal{N}_{\mathbb{C}} \cap W_{r, s}^{\prime}\right) \leq 1
$$


(iii) We have

$$
\mathcal{N}_{\mathbb{C}} \cap V_{r, 0}^{\prime}=\{0\}
$$

Then using Proposition 9.1 of [9], we verify that

$$
\begin{aligned}
& \text { Mult } C_{\gamma_{r, s}}^{\infty}\left(S^{2} T_{\mathbb{C}}^{*}\right)=\operatorname{dim} V_{r, s}+\operatorname{dim} W_{r, s}, \\
& \text { Mult } C_{\gamma_{r, s}^{\prime}}^{\infty}\left(S^{2} T_{\mathbb{C}}^{*}\right)=\operatorname{dim} V_{r, s}^{\prime}+\operatorname{dim} W_{r, s}^{\prime}
\end{aligned}
$$

for all $r, s \geq 0$. From the inclusions (5) and the equalities (6), we obtain directly the following:

Lemma 8. For $r, s \geq 0$, we have

$$
\begin{array}{cc}
\mathcal{C}\left(C_{\gamma_{r, 2 s}}^{\infty}\left(S^{2} T_{\mathbb{C}}^{*}\right)^{\text {ev }}\right)=V_{r, 2 s}, & \mathcal{C}\left(C_{\gamma_{r, 2 s+1}}^{\infty}\left(S^{2} T_{\mathbb{C}}^{*}\right)^{\text {odd }}\right)=V_{r, 2 s+1}, \\
\mathcal{C}\left(C_{\gamma_{r, 2 s}}^{\infty}\left(S^{2} T_{\mathbb{C}}^{*}\right)^{\text {odd }}\right)=W_{r, 2 s}, & \mathcal{C}\left(C_{\gamma_{r, 2 s+1}}^{\infty}\left(S^{2} T_{\mathbb{C}}^{*}\right)^{\text {ev }}\right)=W_{r, 2 s+1}, \\
\mathcal{C}\left(C_{\gamma_{r, 2 s}^{\prime}}^{\infty}\left(S^{2} T_{\mathbb{C}}^{*}\right)^{\text {ev }}\right)=V_{r, 2 s}^{\prime}, & \mathcal{C}\left(C_{\gamma_{r, 2 s+1}^{\prime}}^{\infty}\left(S^{2} T_{\mathbb{C}}^{*}\right)^{\text {odd }}\right)=V_{r, 2 s+1}^{\prime}, \\
\mathcal{C}\left(C_{\gamma_{r, 2 s}^{\prime}}^{\infty}\left(S^{2} T_{\mathbb{C}}^{*}\right)^{\text {odd }}\right)=W_{r, 2 s}^{\prime}, & \mathcal{C}\left(C_{\gamma_{r, 2 s+1}}^{\infty}\left(S^{2} T_{\mathbb{C}}^{*}\right)^{\text {ev }}\right)=W_{r, 2 s+1}^{\prime}
\end{array}
$$

In fact, we have $W_{0,1}=0$ and $W_{0, r}^{\prime}=0$, for $r \geq 0$. Thus by Lemma 8, we see that

$$
C_{\gamma_{0,1}}^{\infty}\left(S^{2} T_{\mathbb{C}}^{*}\right)^{\mathrm{ev}}=\{0\}, \quad \mathcal{N}_{\mathbb{C}} \cap W_{r, 0}^{\prime}=\{0\},
$$

for $r \geq 0$. Since

$$
\operatorname{Mult} \mathcal{N}_{\gamma}^{\text {ev }}=\operatorname{dim}\left(\mathcal{N}_{\mathbb{C}} \cap \mathcal{C}\left(C_{\gamma}^{\infty}\left(S^{2} T_{\mathbb{C}}^{*}\right)^{\text {ev }}\right)\right),
$$

for $\gamma \in \Gamma$, from the equalities (7), Proposition 7 and Lemmas 7 and 8, we deduce Theorem 2 for the quadric $Q_{3}$. We recall that this last result implies both Theorems 1 and 2 .

\section{$\S 6 . \quad$ Integration over flat tori of the quadric}

This section is devoted to some aspects of the proof of Lemma 7 . It is obtained by integrating the symmetric 2-forms of the spaces $V_{r, s}$, $W_{r, s}, V_{r, s}^{\prime}$ and $W_{r, s}^{\prime}$ over flat totally geodesic tori of the quadric $Q_{3}$; for the first equality of Lemma 7 ,(i) we shall also consider the restriction of the operator $D$ to $V_{r, s}$. 
We assume that $X$ is again the quadric $Q_{n}$. The restriction of the mapping $\sigma$ to the subset $\widetilde{Z}_{0}=[0,2 \pi] \times[0, \pi]$ of $\mathbb{R}^{2}$ is a diffeomorphism from $\widetilde{Z}_{0}$ to $Z_{0}$. Therefore if $f$ is a function on $Z_{0}$, then we see that

$$
\int_{Z_{0}} f d Z_{0}=\frac{1}{2} \int_{\widetilde{Z}_{0}}\left(\sigma^{*} f\right)(\theta, \varphi) d \theta d \varphi=\frac{1}{2} \int_{0}^{\pi} \int_{0}^{2 \pi}\left(\sigma^{*} f\right)(\theta, \varphi) d \theta d \varphi
$$

The following formulas relate the decomposition of the bundle $S^{2} T^{*}$ and the parallel vector fields on flat totally geodesic tori and play a fundamental role in our computations of integrals of symmetric 2-forms over these tori. If $J$ is the complex structure of $X$ and $\zeta$ is a vector field on $Z_{0}$, we define complex vector fields on $X$ along $Z_{0}$ by

$$
\zeta^{\prime}=\frac{1}{2}(\zeta-i J \zeta), \quad \zeta^{\prime \prime}=\frac{1}{2}(\zeta+i J \zeta)=\overline{\zeta^{\prime}},
$$

which are of type $(1,0)$ and $(0,1)$, respectively. Let

$$
\begin{gathered}
\pi_{+}: S^{2} T^{*} \rightarrow\left(S^{2} T^{*}\right)^{++} \\
\pi_{++}: S^{2} T^{*} \rightarrow\left(S^{2} T^{*}\right)^{++}, \quad \pi_{+-}: S^{2} T^{*} \rightarrow\left(S^{2} T^{*}\right)^{+-}
\end{gathered}
$$

be the orthogonal projections. If $h$ is a section of $\left(S^{2} T^{*}\right)^{+}$over $X$, we have

$$
\begin{gathered}
\left(\pi_{++} h\right)\left(\xi_{0}, \xi_{0}\right)=h\left(\xi_{0}, \xi_{0}\right), \quad\left(\pi_{++} h\right)\left(\eta_{0}, \eta_{0}\right)=h\left(\eta_{0}, \eta_{0}\right), \\
\left(\pi_{++} h\right)\left(\xi_{0}, \eta_{0}\right)=\left(\pi_{+-} h\right)\left(\xi_{0}, \xi_{0}\right)=\left(\pi_{+-} h\right)\left(\eta_{0}, \eta_{0}\right)=0 \\
\left(\pi_{+-} h\right)\left(\xi_{0}, \eta_{0}\right)=h\left(\xi_{0}, \eta_{0}\right) .
\end{gathered}
$$

If Hess $f$ denotes the Hessian of a complex-valued function $f$ on $X$, because $X$ is a Kähler manifold, from Lemma 1.1 of [7] and (9) we obtain the relations

$$
\left(\pi_{++} \operatorname{Hess} f\right)\left(\eta_{0}, \eta_{0}\right)=2\left(\pi_{+} \operatorname{Hess} f\right)\left(\eta_{0}^{\prime}, \eta_{0}^{\prime \prime}\right)=2(\partial \bar{\partial} f)\left(\eta_{0}^{\prime}, \eta_{0}^{\prime \prime}\right)
$$

and

$$
\begin{aligned}
\left(\pi_{+-} \operatorname{Hess} f\right)\left(\xi_{0}, \eta_{0}\right) & =\left(\pi_{+} \operatorname{Hess} f\right)\left(\xi_{0}^{\prime}, \eta_{0}^{\prime \prime}\right)+\left(\pi_{+} \operatorname{Hess} f\right)\left(\eta_{0}^{\prime}, \xi_{0}^{\prime \prime}\right) \\
& =(\partial \bar{\partial} f)\left(\xi_{0}^{\prime}, \eta_{0}^{\prime \prime}\right)+(\partial \bar{\partial} f)\left(\eta_{0}^{\prime}, \xi_{0}^{\prime \prime}\right) .
\end{aligned}
$$

In $[9, \S 3]$, we give explicit expressions for the vector fields $\xi_{0}^{\prime}$ and $\eta_{0}^{\prime}$ on the intersection of $Z_{0}$ and an open dense subset $V$ of $\mathbb{C} \mathbb{P}^{n+1}$ in terms of a system of holomorphic coordinates on $V$. 
We now again suppose that $X$ is the quadric $Q_{3}$. For $r \geq 0$ and $s \geq 1$, we consider the section

$$
h_{r, s}=\tilde{f}_{r, s-1} \pi_{+-} \operatorname{Hess} \tilde{f}_{0,1}
$$

of $\left(S^{2} T^{*}\right)_{\mathbb{C}}^{+-}$; for $r \geq 0$, we set $h_{r, 0}=0$. For $r, s \geq 0$, we define a subspace $\widetilde{V}_{r, s}$ of $C_{\gamma_{r, s}}^{\infty}\left(S^{2} T_{\mathbb{C}}^{*}\right)$ generated by explicit complex symmetric 2 forms $h_{j}$, with $1 \leq j \leq 7$, which are sections of $\left(S^{2} T^{*}\right)_{\mathbb{C}}^{++}$or of $\left(S^{2} T^{*}\right)_{\mathbb{C}}^{-}$. The subspace $V_{r, s}$ of $C_{\gamma_{r, s}}^{\infty}\left(S^{2} T_{\mathbb{C}}^{*}\right)$ is generated by $\widetilde{V}_{r, s}$ and the section $h_{r, s}$ of $\left(S^{2} T^{*}\right)_{\mathbb{C}}^{+-}$. According to Lemma 3, we have

$$
D h_{r, s}=0 \text {. }
$$

At the end of this section, we shall indicate how to prove the following:

Lemma 9. Let $s \geq 2$ be an even integer and let $r \geq 0$ be an arbitrary integer. Then we have

$$
I\left(h_{r, s}\right) \neq 0 \text {. }
$$

The restriction $D_{r, s}: \widetilde{V}_{r, s} \rightarrow \mathbb{C}^{5}$ of the operator $D$ to the space $\widetilde{V}_{r, s}$ is determined by a $5 \times 7$ matrix, which is given by formula (7.5) of [9]. In $[9, \S 7]$, we use this result to compute the dimension of the space $\widetilde{V}_{r, s}$ and show that the rank of $D_{r, s}$ is equal to $\operatorname{dim} \widetilde{V}_{r, s}-d_{r, s}$ when $s \neq 1$; in particular, when $r, s \geq 2$ we prove that the 2 -forms $\left\{h_{j}\right\}$, with $1 \leq j \leq 7$, are linearly independent and that the mapping $D_{r, s}$ is surjective. Since $\mathcal{N}_{\mathbb{C}}^{e v}$ is contained in the kernel of $D$, from these last results, Lemma 9 and the equality (12), we easily deduce the first equality of Lemma 7,(i).

For $\alpha \in \mathbb{R}$, let $\psi_{\alpha}$ be the element of $S O(5)$ defined by

$$
\begin{gathered}
\psi_{\alpha}(\zeta)_{0}=\sin \alpha \cdot \zeta_{4}+\cos \alpha \cdot \zeta_{2}, \quad \psi_{\alpha}(\zeta)_{4}=\cos \alpha \cdot \zeta_{4}-\sin \alpha \cdot \zeta_{2}, \\
\psi_{\alpha}(\zeta)_{1}=\zeta_{1}, \quad \psi_{\alpha}(\zeta)_{2}=\zeta_{3}, \quad \psi_{\alpha}(\zeta)_{3}=\zeta_{0},
\end{gathered}
$$

where $\zeta \in \mathbb{C}^{5}$.

Let $r \geq 0$ and $s \geq 1$ be given integers. We set

$$
q_{r, s}(t, \theta, \varphi)=\left(t^{2} \cos ^{2} \varphi-\sin ^{2} \theta\right)^{r} \cdot(t \cos \theta \cos \varphi+\sin \theta \sin \varphi)^{s-1}
$$

and

$$
p_{r, s}(t, \theta, \varphi)=(\cos \theta \cos \varphi+t \sin \theta \sin \varphi) \cdot q_{r, s}(t, \theta, \varphi)
$$


for $t, \theta, \varphi \in \mathbb{R}$. We consider the polynomial

$$
P_{r, s}(t)=\int_{\widetilde{Z}_{0}} p_{r, s}(t, \theta, \varphi) d \theta d \varphi
$$

in $t$. Using the expressions for the functions $\psi_{\alpha}^{*} \tilde{f}_{r, s-1}$ and $\psi_{\alpha}^{*} \tilde{f}_{0,1}$ on $V$ and for the vector fields $\xi_{0}^{\prime}$ and $\eta_{0}^{\prime}$ on $Z_{0} \cap V$, according to (12) we verify the equality

$$
\left(\psi_{\alpha}^{*} h_{r, s}\right)\left(\xi_{0}, \eta_{0}\right)(\sigma(\theta, \varphi))=\frac{(-1)^{s}}{2^{r}} p_{r, s}(\sin \alpha, \theta, \varphi),
$$

holds for all $\theta, \varphi \in \mathbb{R}$.

Lemma 10. If $s \geq 2$ is an even integer, then there exists $\alpha_{0} \in \mathbb{R}$ such that the integral

$$
\int_{Z_{0}}\left(\psi_{\alpha_{0}}^{*} h_{r, s}\right)\left(\xi_{0}, \eta_{0}\right) d Z_{0}
$$

does not vanish.

Proof. The coefficient of $t^{2 r+s-1}$ of the polynomial $P_{r, s}(t)$ is equal to the integral

$$
\int_{\widetilde{Z}_{0}} \cos ^{2 r} \varphi(\cos \theta \cos \varphi)^{s-2} \cdot\left((s-1) \sin ^{2} \theta \sin ^{2} \varphi+\cos ^{2} \theta \cos ^{2} \varphi\right) d \theta d \varphi,
$$

which is clearly positive. Thus the polynomial $P_{r, s}$ is non-zero and so there exists a real number $\alpha_{0}$ such that $P_{r, s}\left(\sin \alpha_{0}\right)$ does not vanish. From (8) and (13), we infer that the integral of the lemma corresponding to this element $\alpha_{0} \in \mathbb{R}$ does not vanish.

Since $\psi_{\alpha_{0}}$ induces an isometry of $X$, we see that Lemma 9 is a direct consequence of Lemma 10. The constant term of the polynomial $P_{r, s}(t)$ is easily seen to vanish, and hence so does the integral

$$
\int_{Z_{0}}\left(\psi_{0}^{*} h_{r, s}\right)\left(\xi_{0}, \eta_{0}\right) d Z_{0}
$$

Therefore our proof really does require the variation of the family of integrals

$$
\int_{Z_{0}}\left(\psi_{\alpha}^{*} h_{r, s}\right)\left(\xi_{0}, \eta_{0}\right) d Z_{0}
$$

with $\alpha \in \mathbb{R}$. 
The other assertions of Lemma 7 are proved by performing various integrations over flat tori. They are either done directly or, as in the case of Lemma 9 , by computing the variation of the integrals over a one or two parameter family of flat totally geodesic tori.

\section{References}

[1] M. Berger, "Le spectre d'une variéte riemannienne", Lect. Notes in Math., Vol. 194, Springer-Verlag, Berlin, Heidelberg, New York, 1971.

[2] A. Besse, "Manifolds all of whose geodesics are closed", Ergeb. Math. Grenzgeb., Bd. 93, Springer-Verlag, Berlin, Heidelberg, New York, 1978.

[3] Y. Dieng, Quelques résultats de rigidité infinitésimale pour les quadriques complexes, C. R. Acad. Sci. Paris Sér. I Math., 304 (1987), 393-396.

[4] J. Gasqui and H. Goldschmidt, Déformations infinitésimales des espaces riemanniens localement symétriques. I, Adv. in Math., 48 (1983), 205-285.

[5] — Déformations infinitésimales des espaces riemanniens localement symétriques. II. La conjecture infinitésimale de Blaschke pour les espaces projectifs complexes, Ann. Inst. Fourier (Grenoble), 342 (1984), 191-226.

[6] — Rigidité infinitésimale des espaces projectifs et des quadriques complexes, J. Reine Angew. Math., 396 (1989), 87-121.

[ 7 ] _ On the geometry of the complex quadric, Hokkaido Math. J., 20 (1991), 279-312.

[8] — The infinitesimal rigidity of the complex quadric of dimension four, Amer. Math. J., 116 (1994), 501-539.

[9] _ Radon transforms and spectral rigidity on the complex quadrics and the real Grassmannians of rank two, J. Reine Angew. Math., to appear.

[10] H. Goldschmidt, On the infinitesimal rigidity of the complex quadric, Contemp. Math., 140 (1992), 49-63.

[11] E. Grinberg, Aspects of flat Radon transform, Contemp. Math., 140 (1992), 73-85.

[12] — Flat Radon transforms on compact symmetric spaces with application to isospectral deformations.

[13] V. Guillemin, On micro-local aspects of analysis on compact symmetric spaces, in "Seminar on micro-local analysis", by V. Guillemin, M. Kashiwara and T. Kawai, Ann. of Math. Studies, No. 93, Princeton University Press, University of Tokyo Press, Princeton, N.J., 1979, pp. 79-111.

[14] R. Michel, Problèmes d'analyse géométrique liés à la conjecture de Blaschke, Bull. Soc. Math. France, 101 (1973), 17-69. 
[15] B. Smyth, Differential geometry of complex hypersurfaces, Ann. of Math., 85 (1967), 246-266.

[16] R. Strichartz, The explicit Fourier decomposition of $L^{2}(S O(n) /$ $S O(n-m))$, Can. J. Math., 27 (1975), 294-310.

[17] S. Tanno, Eigenvalues of the Laplacian of Riemannian manifolds, Tôhoku Math. J., 25 (1973), 391-403.

[18] C. Tsukamoto, Infinitesimal Blaschke conjectures on projective spaces, Ann. Sci. École Norm. Sup. (4), 14 (1981), 339-356.

[19] - Spectra of Laplace-Beltrami operators on $S O(n+2) /$ $S O(2) \times S O(n)$ and $S p(n+1) / S p(1) \times S p(n)$, Osaka J. Math., 18 (1981), 407-426.

[20] N. Wallach, "Harmonic analysis on homogeneous spaces", Marcel Dekker, New York, 1973.

J.Gasqui

Institut Fourier,

Université de Grenoble I,

B. P. 74,

38402 Saint-Martin d'Hères Cedex,

France

Jacques.Gasqui@ujf-grenoble.fr

H.Goldschmidt

Department of Mathematics,

Columbia University,

New York, NY 10027,

U. S. A.

hg@math.columbia.edu 